Revista de Matemática: TeOría y Aplicaciones 2018 25(1) : 29-40

CIMPA - UCR ISSN: 1409-2433 (PRINT), 2215-3373 (ONLINE)

\title{
TRATAMIENTO ANALÍTICO DE LA BIFURCACIÓN DE HOPF EN UNA EXTENSIÓN DEL SISTEMA DE LÜ
}

\author{
ANALYTICAL TREATMENT OF THE HOPF \\ BIFURCATION IN AN EXTENSION \\ OF THE LÜ SYSTEM \\ Pablo Emilio CALDERÓN-S AAVEdRA* \\ Evodio Muñoz-Aguirre ${ }^{\dagger}$ JORge Alvarez-MEnA ${ }^{\ddagger}$
}

Received: 19/Sep/2016; Revised: 27/Jun/2017;

Accepted: 16/Dec/2017

Revista de Matemática: Teoría y Aplicaciones is licensed under a Creative Commons Reconocimiento-NoComercial-Compartirigual 4.0 International License.

Creado a partir de la obra en http://www.revistas.ucr.ac.cr/index.php/matematica

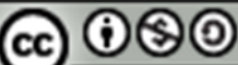

${ }^{*}$ Facultad de Ciencias, Universidad del Tolima, Ibagué, Colombia. E-Mail: pecalderon@ut.edu.co

${ }^{\dagger}$ Facultad de Matemáticas, Universidad Veracruzana, Xalapa, México. E-Mail: evmunoz@uv.mx

${ }^{\ddagger}$ Misma dirección que/Same address as: E. Muñoz-Aguirre. E-Mail: joalvarez@uv.mx 


\title{
Resumen
}

En este artículo se hace un análisis de la bifurcación de Hopf del sistema tridimensional tipo Lorenz introducido por Xianyi Li y Qianjun Ou (2011), este análisis consiste en identificar una región de parámetros del sistema donde la bifurcación de Hopf es no degenerada y supercrítica, aspecto que no es abordado en el artículo de Xianyi Li y Qianjun Ou. Para lograr este objetivo se utiliza el Teorema de la Variedad Central y el Teorema de Hopf. Además, para ilustrar los resultados, se muestran gráficas de algunas trayectorias del sistema, las cuales fueron obtenidas mediante simulación numérica.

Palabras clave: sistema tipo Lorenz; teorema de la variedad central; teorema de la bifurcación de Hopf.

\begin{abstract}
In this paper, we analyze Hopf Bifurcation of the three-dimensional Lorenz-like system introduced by Xianyi Li and Qianjun Ou (2011), this analysis consists of identifying a parameter region, in which the nondegenerate and supercritical Hopf bifurcation occurs, situation that is not discussed by Xianyi Li and Qianjun Ou. To achieve this purpose, we use the Center Manifold Theorem and the Hopf Theorem. In addition, to illustrate the results, the graphics of some trayectories of the system are shown, which were obtained via numerical simulations.
\end{abstract}

Keywords: Lorenz-type systems; center manifold theorem; Hopf bifurcation theorem.

Mathematics Subject Classification: 37G15, 34C23, 34K18.

\section{Introducción}

El sistema de Lorenz fue estudiado por el metereólogo Edward Norton Lorenz cuando investigaba modelos matemáticos para predecir el comportamiento de los gases en la atmósfera, y fue publicado en [15]. Desde entonces han aparecido en la literatura sistemas caóticos con estructura similar al de Lorenz a los que se les ha llamado sistemas Tipo Lorenz: sistema de Chen [2, 5], sistema de Lü [3, 6, 18], Li-Wang [12] y otros [7, 8, 16, 19, 22].

Xianyi Li y Qianjun Ou en [13] estudian el sistema Tipo Lorenz,

$$
\begin{aligned}
\dot{x} & =a(y-x), \\
\dot{y} & =d y-x z, \\
\dot{z} & =g x y-b z+f x^{2},
\end{aligned}
$$


cuando $a>0, f \geq 0, g \geq 0, f+g>0$ y $b, d \in \mathbb{R}$, con vector de estados $(x, y, z) \in \mathbb{R}^{3}$, que en este escrito se denomina sistema de Li-Ou. En [13] destacan aspectos del sistema (1) como la presencia de órbitas caóticas y otras características propias de los sistemas Tipo Lorenz: el número de puntos de equilibrio, la dinámica local, estabilidad en los equilibrios, la existencia de órbitas homoclínicas y heteroclínicas, la presencia de las bifurcaciones de Pitchfork y Hopf, además plantean como problema abierto el estudio de la estructura geométrica del Atractor de Lorenz.

Li y Ou demuestran la existencia de la bifurcación de Hopf en el sistema (1); sin embargo no abordan el análisis de la bifurcación de Hopf, el cual consiste en determinar si la bifurcación es no degenerada, y si es el caso, distinguir si es supercrítica o subcrítica. Este problema ha sido destacado en [12], donde además se menciona que el problema sigue abierto debido su complejidad.

El objetivo de este artículo es hacer un análisis de la bifurcación de Hopf del sistema de Li-Ou (1), cuando el parámetro $f$ toma el valor cero. Para lograr este propósito se utiliza una técnica clásica que hace uso del Teorema de la Variedad Central y del Teorema de Hopf [17], dicha técnica ha sido empleada en [9]. El aporte principal de este trabajo consiste en mostrar analíticamente y con ejemplos la existencia de una región de los parámetros en donde el sistema (1) presenta la bifurcación de Hopf supercrítica. Por otra parte, el sistema en estudio es una extensión del sistema de Lü, pues éste se obtiene de (1) haciendo $f=0$ y $g=1$.

El análisis de la bifurcación de Hopf es de importancia en el estudio de la estabilidad de sistemas dinámicos, pues determina la estabilidad de las órbitas periódicas del sistema (ver [1, 4, 20]). Por otra parte, cuando la estabilidad de una órbita periódica no es la deseada, se usan técnicas para perturbar el sistema con el propósito de cambiar su estabilidad, a lo que se le denomina control de la bifurcación. Es así como el análisis de la bifurcación de Hopf es de utilidad en la teoría del control de la bifurcación (ver [14, 18, 21, 23]).

La estructura del presente artículo es la siguiente. En la sección 2, con el propósito de ofrecer un trabajo autocontenido, se presentan los resultados de [13] sobre la existencia de la bifurcación de Hopf en el sistema de Li-Ou. Para ilustrar los resultados se muestra un ejemplo. En la sección 3 se demuestra que en una región del espacio de parámetros del sistema (1), la bifurcación de Hopf es no degenerada y supercrítica. Los resultados son ilustrados con un ejemplo. Por último en la sección 4 se hace la conclusión del trabajo. 


\section{Propiedades del sistema}

Li y $\mathrm{Ou}$ en [13] encontraron para el sistema (1) que para los parámetros $(a, b, d, f, g)=(10,3,6,1,0)$, el sistema tiene los exponentes de Lyapunov: $\lambda_{L E_{1}}=0.4265, \lambda_{L E_{2}}=0.000001, \lambda_{L E_{3}}=-7.4264$, se observa que dos de estos exponentes son mayores de cero lo que caracteriza la aparición de una órbita caótica. En la Figura 1 se muestra un atractor caótico del sistema, la gráfica corresponde a la órbita con condición inicial $(x, y, z)=\left(\frac{23}{20}, \frac{33}{10}, \frac{7}{2}\right)$.

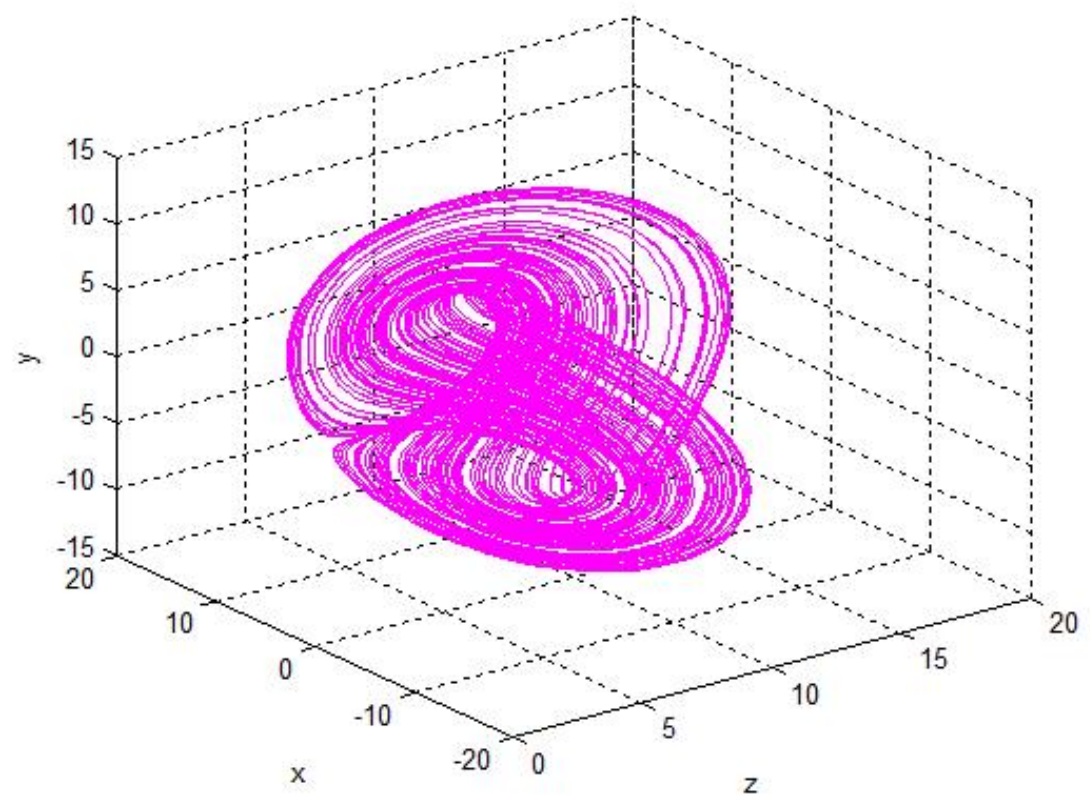

Figura 1: Atractor caótico de Li-Ou.

Los puntos de equilibrio del sistema (1) se clasifican de acuerdo a los siguientes casos:

- Caso $b d<0$ : hay sólo un punto de equilibrio $P_{0}=(0,0,0)$.

- Caso $b d=0$ : si $b=0$ todo punto de la forma $(0,0, z)$ es un equilibrio y para $d=0$ el equilibrio es $P_{0}$.

- Caso $b d>0$ : hay tres puntos de equilibrio $P_{0}=(0,0,0)$,

$P_{1}=\left(\sqrt{\frac{b d}{f+g}}, \sqrt{\frac{b d}{f+g}}, d\right)$ y $P_{2}=\left(-\sqrt{\frac{b d}{f+g}},-\sqrt{\frac{b d}{f+g}}, d\right)$. 
En adelante se restringe el análisis al caso $b d>0$ y $f=0$. Li y Ou en [13] verificaron que en $P_{0}$ el sistema (1) presenta una dinámica sin bifurcación y que en los puntos de equilibrio $P_{1}$ y $P_{2}$ el sistema presenta bifurcación de Hopf, en particular, demostraron que cuando $b>0$ sólo hay un valor crítico de bifurcación,

$$
d_{-}=\frac{a+b}{3}
$$

El siguiente teorema y su demostración pueden consultarse en [13].

Teorema 1 Cuando $a>0, b>0, d>0, f=0$ y $g>0$, las siguientes afirmaciones son válidas para el sistema (1):

1. Cuando $0<d<\frac{a+b}{3}$, los puntos de equilibrio $P_{1}$ y $P_{2}$ son asintóticamente estables.

2. Cuando $\frac{a+b}{3}<d$, los equilibrios $P_{1}$ y $P_{2}$ son inestables.

3. Cuando $d=\frac{a+b}{3}$, en cada equilibrio $P_{1}$ y $P_{2}$ surge una órbita periódica con periodo $T=\frac{2 \pi}{\sqrt{a b}}$.

Por lo tanto el sistema presenta bifurcación de Hopf.

Ejemplo 1 En cada caso del Teorema 1 se presenta un sistema particular y la gráfica de la órbita con condición inicial $\left(x_{0}, y_{0}, z_{0}\right)=\left(\frac{1}{4}, \frac{1}{4}, \frac{89}{100}\right)$.

El sistema con parámetros $(a, b, d, f, g)=\left(\frac{3}{5}, \frac{3}{5}, \frac{3}{10}, 0,2\right)$ satisface las condiciones $a>0, b>0, f=0, g>0 y d<\frac{a+b}{3}$. Entonces, por la parte $1 \mathrm{del}$ Teorema, los equilibrios $P_{1}$ y $P_{2}$ son asintóticamente estables. La Figura 2(a) ilustra el comportamiento de la órbita alrededor de $P_{1}$. Cuando los parámetros son $(a, b, d, f, g)=\left(\frac{3}{5}, \frac{3}{5}, \frac{1}{2}, 0,2\right)$, se satisfacen las condiciones $a>0, b>0$, $f=0, g>0$ y $d>\frac{a+b}{3}$. Luego, la parte 2 del Teorema garantiza que los equilibrios $P_{1}$ y $P_{2}$ son inestables, como se ilustra en la Figura 2(b).

Para los parámetros $(a, b, d, f, g)=\left(\frac{3}{5}, \frac{3}{5}, \frac{2}{5}, 0,2\right)$, se verifican las condiciones de la parte 3 del Teorema: $a>0, b>0, f=0, g>0$ y $d=\frac{a+b}{3}$, por lo que se asegura la existencia de una órbita periódica. La órbita de la Figura 2(c) da evidencia de una órbita periódica.

\section{Análisis de la bifurcación de Hopf}

Esta sección se dedica a demostrar que la bifurcación de Hopf del sistema (1) cuya existencia se afirma en el Teorema 1 es no degenerada y supercrítica. 


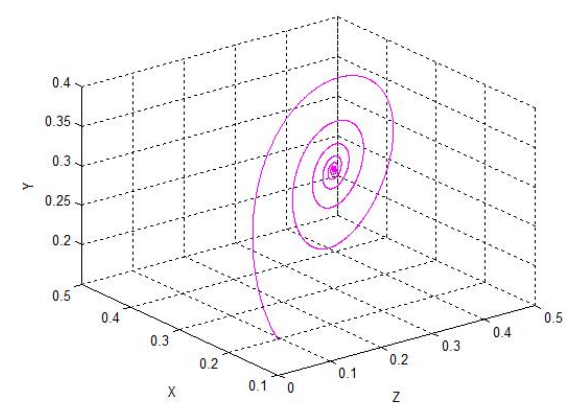

(a) $d=\frac{3}{10}$

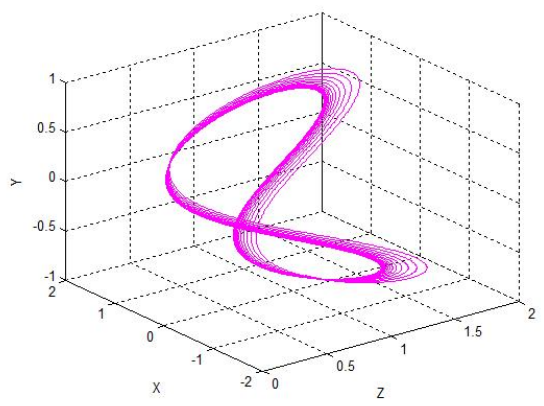

(b) $d=\frac{1}{2}$

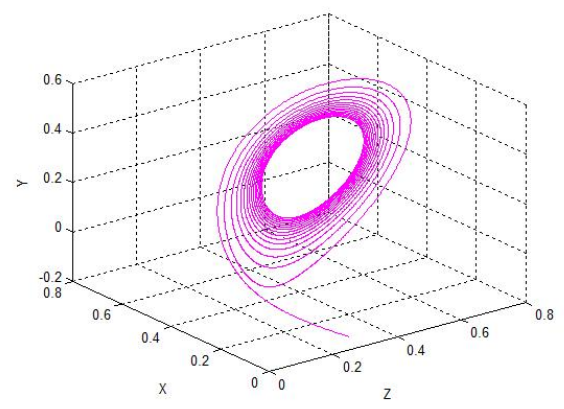

(c) $d=\frac{2}{5}$

Figura 2: Dinámica del sistema 1 con $(a, b, f, g)=\left(\frac{6}{10}, \frac{6}{10}, 0,2\right)$.

Teorema 2 Cuando los parámetros satisfacen $b>0, a=b, d=\frac{a+b}{3}, f=0 y$ $g>0$, el sistema (1) presenta bifurcación de Hopf no degenerada y supercrítica en los puntos de equilibrio $P_{1}$ y $P_{2}$.

Demostración. Bajo las condiciones establecidas en los parámetros, el Teorema 1 garantiza la existencia de la bifurcación de Hopf del sistema en los puntos $P_{1}$ y $P_{2}$. Se demostrará que la bifurcación de Hopf es supercrítica verificando que el primer coeficiente de Lyapunov es negativo en el punto de equilibrio $P_{1}$. Como consecuencia de la simetría del sistema, la conclusión es la misma para el equilibrio $P_{2}$.

La matriz jacobiana $A$ del sistema (1), en el punto $P_{1}$, con las condiciones sobre los parámetros toma la forma,

$$
A=\left(\begin{array}{ccc}
-a & a & 0 \\
-d_{-} & d_{-} & -h \\
g h & g h & -a
\end{array}\right), \text { con } h=\sqrt{\frac{a d_{-}}{g}},
$$


el polinomio característico asociado a la matriz $A$ está dado por,

$$
p(\lambda)=\lambda^{3}+\left(2 a-d_{-}\right) \lambda^{2}+a^{2} \lambda+2 a^{2} d_{-} .
$$

Como consecuencia del Teorema de Hopf y de las condiciones en los parámetros se sigue que $p(\lambda)$ tiene un par de raíces imaginarias conjugadas $\lambda_{1}=\omega i \mathrm{y}$ $\lambda_{2}=-\omega i$. Por otra parte, las raíces del polinomio característico $p(\lambda)$, deben satisfacer las siguientes relaciones:

$$
\begin{aligned}
\lambda_{1}+\lambda_{2}+\lambda_{3} & =-\left(2 a-d_{-}\right), \\
\lambda_{1} \lambda_{2}+\lambda_{2} \lambda_{3}+\lambda_{1} \lambda_{3} & =a^{2} \\
\lambda_{1} \lambda_{2} \lambda_{3} & =-2 a^{2} d_{-} .
\end{aligned}
$$

De (2) se obtiene la tercer raíz $\lambda_{3}=-\frac{4}{3} a$, la cual es negativa dado que $a>0$. Entonces la ecuación (4) se reescribe como

$$
(\omega i)(-\omega i)\left(-\frac{4}{3} a\right)=-\frac{4}{3} a^{3},
$$

y se obtiene $\omega=a$.

Al solucionar el sistema $A q=a i q$, se obtiene el vector propio complejo,

$$
q=\left(\begin{array}{c}
a h \\
a h+a h i \\
a^{2}-\frac{1}{3} a^{2} i
\end{array}\right)
$$

y de la solución del sistema $A v=\lambda_{3} v$, resulta el vector propio real

$$
v=\left(\begin{array}{c}
3 a h \\
-a h \\
-4 a^{2}
\end{array}\right) .
$$

La matriz $P=[\operatorname{Im}(q) ; \operatorname{Re}(q) ; v]$ asociada a los vectores propios y su inversa son:

$$
\begin{aligned}
P & =\left(\begin{array}{ccc}
0 & a h & 3 a h \\
a h & a h & -a h \\
-\frac{1}{3} a^{2} & a^{2} & -4 a^{2}
\end{array}\right), \\
P^{-1} & =\frac{3}{25 a^{2}}\left(\begin{array}{ccc}
\frac{-3 a}{h} & \frac{7 a}{h} & -4 \\
\frac{13 a}{3 h} & \frac{a}{h} & 3 \\
\frac{4 a}{3 h} & \frac{-a}{3 h} & -1
\end{array}\right) .
\end{aligned}
$$

Rev.Mate.Teor.Aplic. (ISSN print: 1409-2433; online: 2215-3373) Vol. 25(1): 29-40, Jan-Jun 2018 
Se traslada el punto de equilibrio $P_{1}=\left(h, h, \frac{2 a}{3}\right)$ al origen mediante la transformación $x=h+y_{1}, y=h+y_{2}, z=\frac{2 a}{3}+y_{3}$, obteniendo:

$$
\dot{Y}=\left(\begin{array}{ccc}
-a & a & 0 \\
-\frac{2 a}{3} & \frac{2 a}{3} & -h \\
g h & g h & -a
\end{array}\right) Y+y_{1} B Y
$$

donde $Y=\left(y_{1}, y_{2}, y_{3}\right)^{T}$ y $B=\left(\begin{array}{ccc}0 & 0 & 0 \\ 0 & 0 & -1 \\ 0 & g & 0\end{array}\right)$.

Para simplificar el sistema (5) se hace el cambio de variable $Y=P Z$, obteniendo el nuevo sistema

$$
\dot{Z}=\left(\begin{array}{ccc}
0 & -\omega & 0 \\
\omega & 0 & 0 \\
0 & 0 & -\frac{4}{3} a
\end{array}\right) Z+a h\left(z_{2}+3 \mu\right) P^{-1} B P Z,
$$

donde $Z=\left(z_{1}, z_{2}, \mu\right)^{T}$. El sistema (6) tiene ahora la forma $\dot{Z}=J Z+F(Z)$, con la matriz de Jordan $J=P^{-1} A P$ y el término no lineal,

$$
\begin{aligned}
F(Z) & =a h\left(z_{2}+3 \mu\right) P^{-1} B P Z \\
& =\frac{-a^{2}\left(z_{2}+3 \mu\right)}{25}\left(\begin{array}{ccc}
1 & 29 & -92 \\
-7 & -3 & -6 \\
\frac{7}{3} & 1 & 2
\end{array}\right)\left(\begin{array}{c}
z_{1} \\
z_{2} \\
\mu
\end{array}\right),
\end{aligned}
$$

además $\left(z_{1}, z_{2}\right)$ pertenece al subespacio central y $\mu$ está en el subespacio estable.

Como consecuencia del Teorema de la Variedad Central, existe $\delta>0$ y una función $H$ en una vecindad del origen $N_{\delta}(0)$, que define la variedad central local $W^{c}(0)=\left\{\left(z_{1}, z_{2}, \mu\right): \mu=H\left(z_{1}, z_{2}\right)\right.$ para $\left.\left\|\left(z_{1}, z_{2}\right)\right\|<\delta\right\}$.

Para el cálculo de la variedad central del sistema (6) se propone la expresión $H\left(z_{1}, z_{2}\right)=a_{1} z_{1}^{2}+a_{2} z_{1} z_{2}+a_{3} z_{2}^{2}$, como en [11, Sec. 5.4]. El cálculo de los coeficientes $a_{1}, a_{2}$ y $a_{3}$ se obtienen de derivar $\mu$ como sigue,

$$
\dot{\mu}=\frac{\partial H}{\partial z_{1}} \dot{z}_{1}+\frac{\partial H}{\partial z_{2}} \dot{z}_{2}
$$

se calculan las derivadas parciales de $H$ y se reemplazan junto con $\dot{z}_{1}, \dot{z}_{2}, \dot{\mu}$ de (6) en (7), se soluciona el sistema resultante y se obtiene

$$
\begin{aligned}
& a_{1}=\frac{42 a^{3}-27 a^{4}}{200\left(4+9 a^{2}\right)}, \\
& a_{2}=\frac{-14 a^{2}+9 a^{3}}{50\left(4+9 a^{2}\right)} \mathrm{y} \\
& a_{3}=\frac{-24 a^{2}-42 a^{3}-27 a^{4}}{200\left(4+9 a^{2}\right)} .
\end{aligned}
$$


Otra consecuencia del Teorema de la Variedad Central es que el flujo en la variedad central está definido por el sistema de ecuaciones diferenciales,

$$
\begin{aligned}
& \dot{z}_{1}=-\omega z_{2}+F_{1}\left(z_{1}, z_{2}\right), \\
& \dot{z}_{2}=\omega z_{1}+F_{2}\left(z_{1}, z_{2}\right),
\end{aligned}
$$

con $\left\|\left(z_{1}, z_{2}\right)\right\|<\delta, F_{1}(0)=F_{2}(0)=0, D F_{1}(0)=D F_{2}(0)=0$, y por $(6)$,

$$
\begin{aligned}
& F_{1}\left(z_{1}, z_{2}\right)=-\frac{a^{2}}{25}\left[z_{1} z_{2}+29 z_{2}^{2}-5 z_{2} H\left(z_{1}, z_{2}\right)+3 z_{1} H\left(z_{1}, z_{2}\right)-276 H^{2}\left(z_{1}, z_{2}\right)\right], \\
& F_{2}\left(z_{1}, z_{2}\right)=-\frac{a^{2}}{25}\left[-7 z_{1} z_{2}-3 z_{2}^{2}-15 z_{2} H\left(z_{1}, z_{2}\right)-21 z_{1} H\left(z_{1}, z_{2}\right)-18 H^{2}\left(z_{1}, z_{2}\right)\right] .
\end{aligned}
$$

Para el cálculo del primer coeficiente de Lyapunov $\ell_{1}$, se usa la fórmula de Guckenheimer [10, Pág. 152]

$$
\ell_{1}=\frac{1}{16}\left(R_{1}+\frac{R_{2}}{\omega}\right)
$$

cuando

$$
\begin{aligned}
R_{1}= & F_{1 z_{1} z_{1} z_{1}}+F_{1 z_{1} z_{2} z_{2}}+F_{2 z_{1} z_{1} z_{2}}+F_{2 z_{2} z_{2} z_{2}}, \\
R_{2}= & F_{1 z_{1} z_{2}}\left(F_{1 z_{1} z_{1}}+F_{1 z_{2} z_{2}}\right)-F_{2 z_{1} z_{2}}\left(F_{2 z_{1} z_{1}}+F_{2 z_{2} z_{2}}\right)-F_{1 z_{1} z_{1}} F_{2 z_{1} z_{1}} \\
& +F_{1 z_{2} z_{2}} F_{2 z_{2} z_{2}} .
\end{aligned}
$$

El primer coeficiente de Lyapunov para el sistema (8) toma la forma,

$$
\ell_{1}(0)=-\frac{142}{65} a^{2}<0
$$

puesto que éste es negativo, como consecuencia del Teorema de Hopf, la órbita periódica es estable.

Dado que en la variedad central el sistema (1) es topológicamente equivalente al sistema (8), la órbita periódica del sistema (1) es estable, por lo tanto la bifurcación de Hopf del sistema de Li-Ou es no degenerada y supercrítica.

Ejemplo 2 Para ilustrar el Teorema 2 se presenta un sistema particular y la gráfica de la órbita con condición inicial $(x, y, z)=\left(\frac{5}{4}, \frac{5}{4}, \frac{89}{100}\right)$. Cuando los parámetros son $(a, b, d, f, g)=\left(\frac{1}{2}, \frac{1}{2}, \frac{1}{3}, 0,2\right)$, se satisfacen las condiciones $b>0, b=a, f=0, g>0 y d=\frac{a+b}{3}$, entonces el Teorema asegura la presencia de una órbita periódica estable. La órbita de la Figura 3 da evidencia de una órbita periódica estable. 


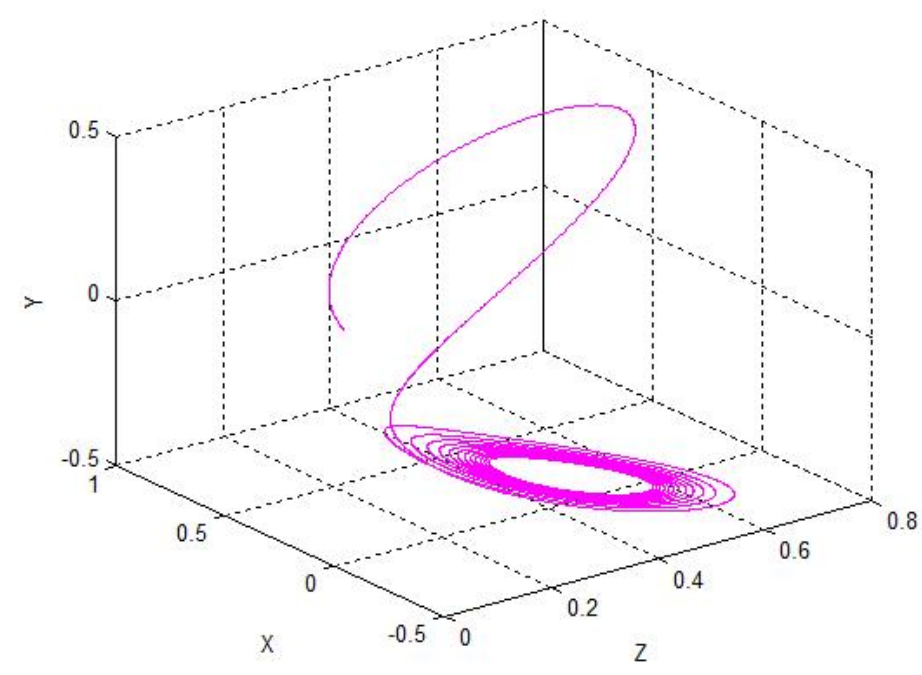

Figura 3: Órbita del sistema (1) con $(a, b, d, f, g)=\left(\frac{1}{2}, \frac{1}{2}, \frac{1}{3}, 0,2\right)$.

\section{Conclusiones}

Se demostró que en los puntos de equilibrio no hiperbólicos el sistema (1) experimenta la bifurcación de Hopf no degenerada y supercrítica en la región de parámetros $a>0, a=b, d=\frac{a+b}{3}, f=0$ y $g>0$. Los resultados de los Teoremas 1 y 2 se ilustraron gráficamente.

Un problema abierto para estudios futuros, es el análisis de la bifurcación de Hopf del sistema (1) en otras regiones de parámetros donde exista esta bifurcación.

\section{Agradecimientos}

A CONACYT por la beca otorgada a Pablo Emilio Calderón Saavedra estudiante del Doctorado en Matemáticas de la Universidad Veracruzana, Xalapa, México.

\section{Referencias}

[1] Algaba, A.; Domínguez, M.; Merino, M.; Rodríguez, A.L. (2015) "Study of the Hopf bifurcation in the Lorenz, Chen and Lü systems", Nonlinear Dyn. 79(1): 885-902. 
[2] Algaba, A.; Fernández-Sánchez, F.; Merino, M.; Rodríguez, A.L. (2014) "Centers on center manifolds in the Lorenz, Chen and Lü systems", Commun. Nonlinear Sciences Numerical Simulation. 19(4): 772-775.

[3] Algaba, A.; Fernández-Sánchez, F.; Merino, M.; Rodríguez, A.L. (2013) "The Lü systems is a particular case of the Lorenz system", Physical Letter A. 377(39): 2771-2776.

[4] Castellanos, V.; Blé, G.; Llibre, J. (2016) "Existence of limit cicles in a tritrophic food chain model with holling functional responses of type I and II", Mathematical Methods in the Applied Sciences 39(1): 1-2.

[5] Chen, C.-T. (1994) System and Signal Analysis. Oxford University Press, New York.

[6] Chen, G.; Lü, J. (2003) "Dynamics of the Lorenz family: analysis, control and synchronization", Chinese Science Press, Beijing 00(1): 0-2.

[7] Chen, G.; Lü, J.; Cheng, D. (2004) "A new chaotic system and beyond: The generalized Lorenz-like system", International Journal of Bifurcation and Chaos 14(5): 1507-1537.

[8] Chen, G.; Oi, G. (2005) "Analysis of a new chaotic system", Physical A: Statistical Mechanics and Its Applications 352(2): 295-308.

[9] Dutta, T.K.; Prajapati, P.; Haloi, S. (2016) "Supercritical and subcritical hopf bifurcations in non linear maps", International Journal of Innovative Research in Technology \& Science 4(2): 14-20.

[10] Guckenheimer, J.; Holemes, P. (1990) "Nonlinear oscillations dynamical systems and bifurcations of vectors fields", Ithaca, Fall.

[11] Kuznetsov, Y. A. (2004) Elements of Applied Bifurcation Theory, third edition. Springer-Verlag, New York.

[12] Li, H.; Wang, M. (2013) "Hopf bifurcation analysis in a Lorenz-type systems", Nonlinear Dynamical 71(1): 235-240.

[13] Li, X.; Ou, Q. (2011) "Dynamical properties and simulation of a new Lorenz-like chaotic system", Nonlinear Dynamical 65(3): 255-270.

[14] Liao, X.; Wang, L.; Yu, P. (2007) Stability of Dynamical Systems. London, Canada. 
[15] Lorenz, E. (1963) "Deterministic nonperiodic flow", J. Atmos. Sci. 20(2): $130-141$.

[16] Pehlivan, I.; Uyaroglu, Y. (2010) "A new chaotic attractor from general Lorenz system family and its electronic experimental implementation", Turk J. Elec. Eng. \& Comp. Sci. 18(2): 171-184.

[17] Perko, L. (2000) Differential Equations and Dynamical Systems, third edition. Springer-Verlag, New York.

[18] Pyragas, K. (2006) "Delayed feedback control of chaos", Phil. Trans. R. soc. A. 364(1846): 2309-2334.

[19] Soon Tee, L.; Salleh, Z. (2013) "Dynamical analysis of a modified Lorenz system", Hindawwi Publishing Corporation Journal of Mathematics 2013(1): 1-8.

[20] Sotomayor, J.; Melo, L.; Braga, D. (2006) Stability and Hopf Bifurcation in the Watt Governor Systems. Cornell University Library, orXiv: Matemáticas/0604177

[21] Tian, Y.-P. (2012) Frequency-Domain Analysis and Desing of Distributed Control Systems. Wiley-IEEE Press.

[22] Yan, Z. (2007) "Hopf bifurcation in the Lorenz-type chaotic system", Chaos, Solitons \& Fractals 31(5): 1135-1142.

[23] Yu, P.; Lü, J. (2010) "Bifurcation control for a class of Lorenz-like systems", International Journal of Bifurcation and Chaos 21(9): 2647-2664. 